\title{
A new method using medians to calibrate single-parameter spatial interaction models
}

\author{
Louis A. Merlin \\ Florida Atlantic University \\ Imerlin@fau.edu
}

\begin{abstract}
I present a method for calibrating the impedance parameter of a gravity spatial interaction model using only the median travel time as a measure of observed traveler behavior. Complete information about the spatial structure of origins, destinations, and travel times between origins and destinations is also required. Using Monte Carlo simulation techniques on stylized cities, I attempt to recover true (a priori known) impedance values with this method for a range of impedance values for both negative exponential and power impedance functions. The results are compared with estimates obtained by other fast methods. The proposed method proves to provide a fairly accurate estimate of the impedance parameter, with a mean percent error typically below $20 \%$ and often below $10 \%$ for common impedance values. The proposed method is an improvement over existing calibration methods in several respects. First, it allows for the estimation of the impedance parameter directly without lengthy iterative calculations. Second, because it only requires median travel times, it can be calibrated with smaller samples $(\mathrm{n} \sim 200)$, allowing the construction of gravity models for specific modes and/or travel purposes. And third, the method does not require expensive travel demand software and so can be implemented more widely in practice.
\end{abstract}

\author{
Article history: \\ Received: May 21, 2019 \\ Received in revised form: \\ November 14, 2019 \\ Accepted: November 17, 2019 \\ Available online: January 29, \\ 2020
}

\section{Introduction}

Spatial interaction models are mathematical models that help describe or explain the likelihood of interactions across space. They are premised upon the idea that interactions between more distant locations are less likely, in many cases because the cost of such interaction varies with distance. Geographers, sociologists, demographers, and urban planners employ spatial interaction models to explain activities such as migration, travel behavior, and social interactions. In particular, transportation analysts frequently apply spatial interaction models to understand travel behavior, such as the factors that underly trip distribution for a specified trip purpose.

Spatial interaction models can take on different mathematical forms that account for the various factors that influence spatial interactions. Perhaps the most commonly used spatial interaction model is the gravity or potential model, which assumes that interactions are less likely over greater distances (or costs) and are more likely between larger centers of activity, in an analogy to the physical force of gravity

Copyright 2020 Louis A. Merlin

http://dx.doi.org/10.5198/jtlu.2020.1614

ISSN: 1938-7849 | Licensed under the Creative Commons Attribution - Noncommercial License 4.0

The Journal of Transport and Land Use is the official journal of the World Society for Transport and Land Use (WSTLUR) and is published and sponsored by the University of Minnesota Center for Transportation Studies. 
between spatial bodies (Hayes \& Wilson, 1971). The intervening opportunities model also takes into account the spatial locations of destinations, but spatial distance has no fixed effect within the intervening opportunities model; rather closer destinations are more likely to be selected over more distant ones, and it is these intervening opportunities that make more distant destinations less attractive (Hayes \& Wilson, 1971; Stouffer, 1940). Another related spatial interaction model is the radiation model, which assumes that the interaction between two centers is directly proportional to their size but inversely proportion to the number of intervening opportunities, i.e., the number of alternative destinations that are the same distance or closer to the proposed destination (Piovani, Arcaute, Uchoa, Wilson, \& Batty, 2018; Simini, González, Maritan, \& Barabási, 2012).

The measurement of accessibility, often defined as the ease of access to destinations of interest, necessarily rests upon a model of spatial interaction. This is because understanding the "ease of access" requires an understanding of the degree to which the costs or impedance of travel inhibits interactions over distance. Therefore, the accurate calculation of accessibility requires a spatial interaction model that describes to what degree distance presents an obstacle to interactions over space. The most commonly employed accessibility measure among practitioners is the cumulative opportunities model (Boisjoly \& El-Geneidy, 2017b; Gutman \& Tomer, 2016), which assumes a binary structure to space, i.e., either a destination is close enough to be of interest or it is not. The gravity-based measure is the second most common type of accessibility measure; however, the calibration of gravity-based spatial interaction models presents an obstacle to many researchers and practitioners interested in accessibility analysis. In accessibility analysis, it is not uncommon for the impedance to be assumed to be a constant value, or for the analyst to use a streamlined method such as calibrating the impedance to the trip length distribution. All of this points to the need for straightforward and streamlined methods for the accurate calibration of gravity-based spatial interaction models.

The proposed method in this paper is superior to existing calibration methods for several reasons. The first is that the traditional complex, iterative calibration required for the doubly-constrained spatial interaction models can be avoided (Fotheringham \& O'Kelly, 1989; Williams, 1976). An accurate estimate of the impedance parameter can be derived quickly via the proposed method. If a full doubly-constrained spatial interaction model is desired, then the inverse balancing factors only need be calibrated a single time based upon this impedance estimate.

The second advantage of the proposed method is that because the calibration of the impedance parameter requires only an accurate median cost as an input, calibration is possible with smaller data samples. Therefore, analysts can readily derive impedance parameters for particular population segments, modes, or trip purposes, opening the world of accessibility analysis to a wider range of applications beyond job accessibility. For example, separate impedance parameters can be derived for work and non-work travel, or even for specific kinds of non-work travel such as social and recreational travel.

Thirdly, the proposed method can be implemented without expensive travel demand modeling software. The following implementation was conducted in free, open-source $\mathrm{R}$ software, and implementation of the proposed method with commonly available spreadsheet software is likely feasible.

Finally, the method rests upon a highly intuitive principle, which may be an effective basis for further innovation. That principle is the following - that the median travel time should be the one where the total destination opportunity (accessibility) passed is equivalent to the total destination opportunity not yet reached. By marrying a gravity model to the principle of opportunities passed, this brings the gravity model closer to the conceptually distinct intervening opportunities model (Goncalves \& Ulyssea-Neto, 1993; Lenormand, Bassolas, \& Ramasco, 2016).

The organization of the remaining paper is as follows. First, the basic form of gravity-based spatial interaction models is discussed. The following section reviews the many challenges to the calibration of spatial interaction models, including the selection of a zonal structure, the issue of self-potential, the se- 
lection of a functional form, and methods of calibration. Median calibration methods are also discussed at the end of this section. The newly proposed method is then detailed in a step-by-step fashion in the following section. Then follows a case study where the method is tested for both negative exponential and power functions and its accuracy is compared with alternative fast methods. Next comes a discussion section that addresses how the method could be applied and why some of the estimated impedance parameters are further off than others. The conclusion section reiterates the proposed method, the case study analysis, and the findings in brief form.

\section{The basic form of gravity-based spatial interaction models}

Spatial interaction models can be unconstrained, production-constrained, attraction-constrained, or doubly-constrained (See equations 1-4) (Fotheringham \& O'Kelly, 1989). Unconstrainted models predict flows based on the mass of source activity at the origin, the mass of attraction activity at the destination, and the impedance between the origin and destination (equation 1). Production-constrained models are similar to unconstrainted models, but the total flows leaving each origin zone are known and therefore used as a constraint in calibration (equation 2). Attraction-constrained models likewise have known total flows into each destination zone, which are in turn used as a constraint (equation 3). Doubly-constrained models have both known total flows from each origin and to each destination, and both known quantities are then used to constrain the model and obtain a more accurate estimate of spatial interaction behavior (equation 4). These four types of models are reviewed in detail in Fotheringham and O'Kelly (1989) and by Wilson (1971).

$$
\begin{aligned}
& T_{i j}=O_{i} D_{j} f\left(c_{i j}\right) \\
& T_{i j}=A_{i} O_{i} D_{j} f\left(c_{i j}\right) \\
& T_{i j}=B_{j} O_{i} D_{j} f\left(c_{i j}\right) \\
& T_{i j}=A_{i} B_{j} O_{i} D_{j} f\left(c_{i j}\right)
\end{aligned}
$$

In these formulas, $T_{i j}$ is the predicted flow from zone $i$ to zone $j$. $O_{i}$ is the number of travelers living in zone $i, D_{\mathrm{j}}$ is the number of destinations located in zone $j, c_{i j}$ is the cost of travel from zone $i$ to zone $j$ and $f()$ is the impedance function, which transforms a positive cost $c_{i j}>0$ into an impedance factor that is non-increasing, i.e., that decreases as the cost of travel increases.

\section{Challenges in the calibration of spatial interaction models}

\subsection{Zonal structure and sampling}

Spatial interaction models typically impose a zonal structure in order to avoid the informational complexity and data requirements of considering every building being a possible origin and destination. However, there is little guidance available on what the most appropriate zonal structure should be. Different levels of spatial aggregation can produce differing results, per the Modifiable Areal Unit Problem (MAUP) (Batty \& Sikdar, 1982; Horner \& Murray, 2002). At first blush, it might seem that a greater number of zones is preferable because smaller zones provide greater accuracy in the calculation of zoneto-zone travel costs. However, as the number of zones increases, any non-complete sample becomes an increasingly inaccurate representation of the proportion of flows between specific pairs of zones. In a 
system with $n$ zones, the number of potential flows is $n^{\wedge} 2$, so there is a high likelihood that at least some of these zone-to-zone sample estimates are inaccurate if the number of zones is large and the sample size is small.

Maximum likelihood methods work well with near-complete data, but in many cases only a sample of total travel behavior is available. As mentioned above, with sample data, many zone-to-zone flows in the sample may highly biased, or worse still, some zone-to-zone flows will appear as 0 (Ortuzar \& Willumsen, 2011). Since all four spatial-interaction model types are multiplicative, this creates a particular problem - for a particular OD pair between origin i and destination $\mathrm{j}$, if the observed sample shows a flow of 0 between $i$ and $j$ but the number of origin travelers and destinations is non-zero, then one of the two constraints $\mathrm{Ai}$ or $\mathrm{Bj}$ is forced to take on a zero value. If, for example, the destination $\mathrm{j}$ takes on a $B j=0$, then the spatial interaction model will predict 0 trips to that destination from all origins. The problem of 0 or small flows between zones presents a barrier to other forms of model calibration as well (Gray \& Sen, 1983).

Murat (2010) tests a range of sample sizes for calibrating doubly-constrained power and exponential models of four trip types using car-based travel times from the 2006 Istanbul travel survey. He finds that samples as small as 1000 produce similar root mean square error predictions of mean travel time and trip length distribution as using the full data. He handles the problem of zero flows by substituting the Furness Biproportional Balancing Procedure for the traditional iterative procedure of calculating balancing factors (Dennett, 2012).

One method of addressing the problem of zero flows is to fit an origin-constrained spatial interaction model to sample data, only including those origins that have non-zero trip counts. The disadvantage of this approach is that this type of spatial interaction model is known to be less accurate than the doubly-constrained model, and such a model can contain bias if it does not account for competition among destinations (Fotheringham, 1983).

Such singly-constrained models can often be calibrated accurately with sample data using maximum likelihood methods. However, since there is a constraint that must be estimated for each origin in the sample, this can greatly reduce the effective degrees of freedom and therefore the effective sample size for estimating impedance parameters. Take for example the 2017 Southeast Florida Household Travel Survey (WSP Parsons Brinckerhoff, 2017). This survey included 2,096 households and 19,630 trips. To calibrate home-to-work commuting trips by transit, there are a total of just 263 such trips coming from 122 origins. The sample size is sufficiently large for maximum likelihood estimation of the required 123 parameters (122 origin constraints +1 impedance parameter), but barely so.

An additional issue with the calibration of spatial interaction models from sample data is that weights are set to reflect a representative population and not a representative set of trips. Therefore, if a person with a high population weight takes a trip of unusual length, this trip will be over-represented in the sample and could throw off the impedance estimate.

\subsection{Self-potential or intrazonal trips}

Another concern is how to accurately estimate intrazonal costs, i.e., the cost of travel from an origin within a zone to a destination within the same zone. Melhorado, Demirel, Kompil, Navajas, and Panayotis (2016) test four different measures of self-potential: area-based, fixed values, density-based and point-to-point. They find that fixed values and area-based measures tend to underestimate true intrazonal travel times for their data on travel between 23 European capital regions. Point-to-point estimation of destinations within each zone provides the most accurate results. The inaccurate estimation of internal travel times can have a large influence on the measurement of accessibility if zones are large in terms of the number of destinations they contain. Stepniak and Jacobs-Crisioni (2017) suggest using mean distance to the population centroid as a fast method to estimate the cost of intrazonal trips. 
Another solution to the problem of self-potential is to ignore intrazonal trips in the calibration of the impedance function (Lenormand et al., 2016; Ortuzar \& Willumsen, 2011). However, for the purposes of calculating accessibility, intrazonal potential cannot be ignored and an intrazonal cost must be introduced.

\subsection{Functional form}

The most commonly used functional forms for the cost element of spatial interaction models are negative exponential (equation 5), power functions (equation 6), and combined function that include both exponential and power elements (equation 7). The combined exponential-power functional form is also called the Tanner function. Other functional forms that have been explored include the logistic function (equation 8) (de Vries, Nijkamp, \& Rietveld, 2009) and the log-normal function (equation 9) (Feldman, Forero-Martinez, \& Coombe, 2012; Reggiani, Bucci, \& Russo, 2011; Taylor, 1971). An alternative to the spatial interaction models is the intervening opportunities model, which considers the availability of more proximate alternatives to be the determinative factor in destination choice rather than travel costs (Ortuzar \& Willumsen, 2011). Goncalves and Ulyssea-Neto (1993) develop a combined intervening opportunities-gravity model and calibrate it to public transport trips in Santa Catarina, Brazil. This model potentially offers the strengths of taking into account both travel costs and the relative level of accessibility of competing destinations.

$$
\begin{aligned}
& f\left(c_{i j}\right)=e^{-\beta c i j} \\
& f\left(c_{i j}\right)=c_{i j}^{-\alpha}=e^{-\alpha \ln \left(c_{i j}\right)} \\
& f\left(c_{i j}\right)=c_{i j}^{-\alpha} e^{-\beta c i j} \\
& \ln \left[f\left(c_{i j}\right)\right]=\gamma_{1} /\left[1+\left(\frac{c_{i j}}{\gamma_{2}}\right)^{\gamma^{3}}\right] \\
& f\left(c_{i j}\right)=\frac{1}{c_{i j} * \sigma * \sqrt{2} \pi} e^{-\frac{\left(l n c_{i j}-\mu\right)}{2 \sigma^{2}}}
\end{aligned}
$$

Lenormorand et al. (2016) compare gravity, intervening opportunity, and radiation models to explain commuting trip distribution, integrating data from six countries and two cities into their analysis. They find that gravity models explain commuting patterns most accurately. Further, they find that the negative exponential function works best in most cases, though such functional forms do not accurately capture relative sparse long-distance commuting flows. De Vries et al. (2009) examine commuting between cities in Denmark, using the financial cost of commuting by car as their measure of impedance and find that the logistic function performs better than the exponential and power functions. In particular, they find that the marginal cost has a decreasing effect on destination attractiveness past a point of inflection as determined by the logistic function. Reggiani et al. (2011) find that log-normal and power functions better fit Germany-wide commuting patterns than does the negative exponential. Fotheringham and O'Kelly (1989) recommend negative exponential function for shorter trips and the power function for longer trips. 


\subsection{Calibration}

Calibration of the doubly-constrained spatial interaction model remains complex, though many alternative techniques have been proposed. The basic problem is the interdependence of the $A_{i}$ values, the $B_{\mathrm{j}}$ values, and the impedance parameters within the impedance function (Fotheringham \& O'Kelly, 1989, p. 53). Once the impedance parameters of $f()$ are known, then $A i$ and $B_{\mathrm{i}}$ can be calculated iteratively through the formulas below:

$$
\begin{gathered}
A_{i}=1 / \sum_{j} B_{j} D_{j} f\left(c_{i j}\right) \\
B_{j}=1 / \sum_{i} A_{i} O_{i} f\left(c_{i j}\right)
\end{gathered}
$$

But likewise, the impedance parameters within the impedance function $f()$ cannot be known until the balancing factors are determined.

The practical suggestion of Ortuzar and Willumsen (2011) is to first estimate the balancing factors through Furness balancing procedure and then estimate the impedance parameters taking these balancing factors as fixed.

A fast method for estimating single-parameter impedance functions (either the exponential or power functions in equations 5 or 6 above) given a set of fixed balancing factors is due to Hyman (Batty \& Mackie, 1972; Hyman, 1969). The essential motivation for this method comes from the fact that the average cost for the estimated flows must be equal to the average cost for the observed flows. This constraint is expressed as:

$$
c^{f}=\sum_{i j} T_{i j} f\left(c_{i j}\right) / \sum_{i j} T_{i j}=\sum_{i j} N_{i j} f\left(c_{i j}\right) / \sum_{i j} N_{i j}=c^{*}
$$

Where $T_{i j}$ represents estimated flows from the spatial interaction model, and $N_{i j}$ represents observed flows from the sample. This means that the left-hand side represents the average estimated trip cost due to the current form of the impedance function $\mathrm{f}$, while the right-hand side represents the observed average trip cost from the sample $c^{*}$. Details for following this procedure are produced in Ortuzar and Willumsen (2011, p. 193). In successive steps the parameter is adjusted until cf converges in value to $c^{*}$.

To be precise, once the impedance parameter is calculated through the Hyman method and the impedance function $f()$ is set, the inverse balancing factors should again be re-calibrated by employing equations 10 and 11 iteratively until a new balancing factor convergence is achieved. However, the practical value of this recalibration may be minimal; it is unclear from the literature if this is a necessary step or how much additional accuracy could be obtained by this recalibration.

When the impedance function has multiple parameters, first balancing factors are estimated, such as through Furness balancing procedure, and then a maximum likelihood model is estimated to determine the parameters of the impedance function.

Several other methods have been proposed for calibrating the numerous and mutually dependent parameters of the doubly-constrained spatial interaction model. Williams (1976) compares three methods for calibrating single-parameter impedance functions - methods due to Hyman, Evans, and Hathaway. Williams finds that all such methods work quickly but that Hyman's method provides the greatest precision and is the most straightforward to implement. Sen and Pruthi (1983) propose a fast, weighted-least-squares method that works only when intrazonal flows are not accounted for. Diplock and Openshaw (2010) explore the use of genetic algorithms to calibrate production-constrained, nega- 
tive exponential models and find that such strategies work reliably but take longer to converge than quasi-Newton methods. De Vries et al. (2009) use non-linear weighted least squares on log-transformed equations to calibrate exponential, power, and logistic-based models and include the numerous origin and destination constants (balancing factors) within their model calibration. Other non-linear methods have also been applied, such as Hooke and Jeeve's discrete step method (Goncalves \& Ulyssea-Neto, 1993). Step-wise methods that search for the best parameter values over a constrained range are also not uncommon (Feldman et al., 2012; Murat, 2010).

As an aside, it is important to note that the observed trip length distribution is often the object taken to be fitted to (Feldman et al., 2012; Murat, 2010; Ortuzar \& Willumsen, 2011; Piovani et al., 2018). However, I have not been able to find a rigorous mathematical basis for directly fitting the impedance function to the observed trip length distribution. Fitting directly to a trip length distribution is not equivalent to fitting a spatial interaction model, because the spatial structure of origins and destinations is not accounted for and therefore is likely to result in an incorrect calibration of the impedance function (Krizek, 2010).

\subsection{Median calibration methods}

Osth, Reggiani, and Galiazzo (2014) proposed a method of calibration based only upon the median travel time, with the goal of avoiding the modifiable areal unit problem (MAUP). If spatial impedance could be determined without reference to a specific zonal structure, the spatial model would presumably be more robust and generalizable. Their proposed method of median calibration is termed a "half-life" method and is based on the concept of radiation half-life in physics. The premise of this method is that the median commute corresponds to the commute duration of half the population, and therefore it represents the point at which half of the traveling population has "decayed" or dropped off from the original total population, having already found a suitable destination by this time. Based on this theory, Osth et al. (2014) estimate the gravity impedance parameter as follows:

$$
\beta=\ln (0.5) / \mathrm{mdn}
$$

where $m d n$ is the median commute time in minutes.

While an ingenious method, this method of calibration based upon median travel times suffers from two flaws. First, it does not consider the spatial structure of the geography being analyzed and so the half-life principle is not justified conceptually. A rate of decay proportional to the amount of substance remaining makes sense for a physically radioactive entity. But the decay rate for commuters must correspond in some sense to the presence of available destinations that can be reached at any given point in time. The half-life of commuters as proposed is only analogous to the physical case of radiation if the number of destinations reachable within each minute is proportional to the number of remaining travelers, which is unlikely to be the case. The second flaw in the proposed method is that it produces inaccurate results. Osth et al. (2014) report an R-square value of $\sim 0.22$ for the half-life calibrated model versus an R-square of -0.95 for the doubly-constrained model in terms of describing commuting flows across Swedish municipalities in 1995 and 2008. Although the approach is novel, it is not sufficiently accurate for general use.

The method proposed herein is inspired by the half-life method of Osth et al. (2014) but addresses its greatest flaws. It explicitly accounts for the spatial structure of the geography of analysis in the calculation of the impedance parameter, and it can produce quite accurate results, often estimating the impedance parameter within $10 \%$ of the true value. It leverages the insights of the Osth et al. (2014) paper that the median commute is a suitable data point for calibrating the single unknown parameter in either the exponential or power versions of the gravity formula. 
How should the half-life concept be applied to spatial interactions? The median travel time is conceptually important in a spatial context. It indicates the point at which exactly half the travelers have reached their destination. Therefore, at the median travel time, the amount of destination opportunity passed should be equal to the amount of destination opportunity remaining. This means that for the average traveler, the accessibility reachable by the median travel time should be equal to the accessibility reachable by more than the median travel time. This balance in the amount of accessible opportunity before and after the median travel time is expressed in equation 14 below, where med is the median travel time, $\delta_{t}$ is the attractiveness of destinations located distance $t$ from all origins and $f(c)$ is the impedance due to cost $c$. In that sense, the half-life principle is here retained.

$$
\sum_{t \leq \text { med }} \delta_{t} * f(c)=\sum_{t \geq m e d} \delta_{t}^{*} f(c)
$$

\section{$4 \quad$ Method}

This section illustrates the procedure for calibrating the impedance parameter given the median travel time (or cost) for a particular population segment, mode, travel purpose, and time of day traveling between a known set of origins and destinations. The data requirements for the procedure are as follows:

- Median travel time (or cost)

- Counts of the population in each zone

- Counts or measures of destinations (or destination attractiveness) in each zone

- Travel times (or costs) between each origin and each destination for the given mode and the given time of day

To illustrate the method, some mathematical notation is helpful.

The median travel time is denoted $m d n$.

Let the zones $z$ be enumerated $i$ or $j=1,2,3, \ldots, \mathrm{N}$ where $N$ is the total number of zones.

The population/origin count for each zone $i$ is indicated by $o i$.

The destination count for each zone $i$ is indicated by $d_{i}$.

The travel time between each origin $z_{i}$ and destination $z_{j}$ is indicated by $t_{i j}$.

Let the travel time $t$ be denominated in minutes (positive integers) where $t=1,2,3, \ldots \mathrm{T}$ and $T$ is the longest possible travel time across any pair of zones in the city $z_{i}$ and $z_{j}$

Step 1: Create a table of the number of destinations $D_{i t}$ reachable for every minute $t=1,2,3, \ldots, \mathrm{T}$ from each origin zone $z_{i}$.

Create a table $\tau$ of the marginal number of destinations reachable by minute from each origin zone. That is, leaving from each origin $i$, the figure in each cell of the table will be the total number of destinations reachable from $i$ that are reached in the tth minute, but not in the $t-1$ st minute. The origins range from $1, \ldots, \mathrm{i}, \ldots, \mathrm{N}$ across all origins, and the travel times $\mathrm{t}$ range from $1,2, \ldots \mathrm{T}$ minutes, where $T$ is the largest travel time possible in the zonal system. 
This table $\tau$ appears as follows:

\begin{tabular}{|c|c|c|c|c|c|c|c|}
\hline $\begin{array}{l}\text { Columns: Minutes } \\
\text { Rows: Origins Zones }\end{array}$ & 1 & 2 & $\ldots$ & $\ldots \mathrm{t} \ldots$ & $\cdots$ & $\mathrm{T}-1$ & $\mathrm{~T}$ \\
\hline Zone 1 & $\mathrm{D}_{11}$ & $\mathrm{D}_{12}$ & $\ldots$ & $\mathrm{D}_{1 \mathrm{t}}$ & $\ldots$ & $\mathrm{D}_{1, \mathrm{~T}-1}$ & $\mathrm{D}_{1, \mathrm{~T}}$ \\
\hline Zone 2 & $\mathrm{D}_{21}$ & $\mathrm{D}_{22}$ & $\ldots$ & $\mathrm{D}_{2 \mathrm{t}}$ & $\ldots$ & $\mathrm{D}_{2, \mathrm{~T}-1}$ & $\mathrm{D}_{2, \mathrm{~T}}$ \\
\hline$\ldots$ & & & $\ldots$ & & $\ldots$ & & $\ldots$ \\
\hline i & & & & $\begin{array}{l}\mathrm{D}_{\mathrm{it}}= \\
\text { Sum of } \mathrm{d}_{\mathrm{j}} \text { reachable from } \\
\text { zone } \mathrm{i} \text { in } \mathrm{t} \text { minutes } \\
\text { (not cumulative) }\end{array}$ & & & \\
\hline$\ldots$ & & & & & & & $\ldots$ \\
\hline Zone N-1 & $\mathrm{D}_{\mathrm{N}-1,1}$ & $\mathrm{D}_{\mathrm{N}-1,2}$ & $\ldots$ & $\mathrm{D}_{\mathrm{N}-1, \mathrm{t}}$ & $\ldots$ & $\mathrm{D}_{\mathrm{N}-1, \mathrm{~T}-1}$ & $\mathrm{D}_{\mathrm{N}-1, \mathrm{~T}}$ \\
\hline Zone $\mathrm{N}$ & $\mathrm{D}_{\mathrm{N}, 1}$ & $\mathrm{D}_{\mathrm{N}, 2}$ & $\ldots$ & $\mathrm{D}_{\mathrm{N}, \mathrm{t}}$ & $\ldots$ & $\mathrm{D}_{\mathrm{N}, \mathrm{T}-1}$ & $\mathrm{D}_{\mathrm{N}, \mathrm{T}}$ \\
\hline
\end{tabular}

In general, the mathematical formula for each element of this table from the $i_{\text {th }}$ row (zone) and the $t_{t h}$ column (minute) is the following:

$$
D_{i t}=\sum_{j=1 \ldots J, t-1<t_{i j} \leq t} d_{j}
$$

Step 2: Create an array of the average number of destinations reachable from all origins for every minute $\mathrm{t}=1,2,3, \ldots, \mathrm{T}$.

Create an array $\delta$ describes the spatial structure of reachable destinations for the average traveler in the environment under analysis.

Weight each row in the above table by the population at each origin, and then take the weighted sum.

This array $\delta$ appears as follows:

\begin{tabular}{|l|l|l|l|l|l|}
\hline Minutes & 1 & 2 & $\ldots$ & $\mathrm{T}-1$ & $\mathrm{~T}$ \\
\hline All Zones & $\delta_{1}$ & $\delta_{2}$ & $\begin{array}{l}\delta_{\mathrm{t}}=\text { Population-weighted average total destinations } \mathrm{d} \\
\text { reachable from all zones in t minutes }\end{array}$ & $\delta_{\mathrm{T}-1}$ & $\delta_{\mathrm{T}}$ \\
\hline
\end{tabular}

The mathematical formula for each array member $\delta_{\mathrm{t}}$ is:

$$
\delta_{t}=\left(\sum_{i=1}^{N} o_{i} * D i t\right) /\left(\sum_{i=1}^{N} o_{i}\right)
$$

Step 3: Determine the impedance coefficient that balances the attractiveness of destinations reached within the median time with the attractiveness of destinations reached at longer than the median travel time.

This is the core insight of the process: That the median travel time should exactly balance the amount of opportunity passed with the amount of opportunity not yet reached.

Mathematically, this is determined by minimizing the objective function of the difference between the amount of opportunity reached and the amount not yet passed. The estimated impedance $\left(\beta_{\text {MED }}\right)$ is determined by minimizing this difference: 


$$
\operatorname{Min}\left(\operatorname{abs}\left(\sum_{t=1}^{m d n} \delta_{t} * e^{\beta_{M E D * t}}-\sum_{t=m d n+1}^{T} \delta_{t} * e^{\beta_{M E D * t}}\right)\right)
$$

The impedance value $\beta_{M E D}$ that brings the above term as close to 0 as possible is the estimated impedance.

Practically, this can be achieved by a hill-climbing (descending) procedure that tests different values of $\beta_{M E D}$ until equation (17) is minimized. Because the right-hand side of equation (17) decreases more quickly than the left-hand side, equation (17) only has no local minima and the global minimum will be found.

Note that although the negative exponential form of the impedance function is utilized here, any single-parameter impedance function could be calibrated via a similar objective function. The concept of half-life is generalizable to power-based as well as exponential impedance functions.

\section{$5 \quad$ Case study with simulated city data}

I built a series of simulated cities with commuting patterns of workers and jobs to test the above procedure. These simulated cities are not intended to mimic the land-use patterns of real-world cities but are merely intended as a challenging test-bed for the proposed calibration method. Each city is a $20 \mathrm{x} 20$ grid of zones (See Figure 1 below). Each zone can be indicated by a coordinate $(x, y)$ where $x$ corresponds with the zone's column number and $y$ corresponds with the zone's row number. The total number of workers and jobs in the city are both fixed at 400,000. The number of workers for each zone is drawn from a Normal distribution with a mean of 1,000 and a standard deviation of 300 , with the city-wide total normalized to 400,000. The employment within each zone is drawn from an Exponential distribution with a mean of 1,000. An Exponential distribution better simulates the more concentrated nature of employment in cities, which tends to be high in a few select areas and low in most residential areas. The concentration of employment also serves as a kind of "stress test" for the spatial interaction model because feasible destinations are not nearby for every origin. Zonal employment is likewise adjusted to ensure a citywide total of 400,000. Figure 2 illustrates worker population and employment densities by zone for a sample city. 


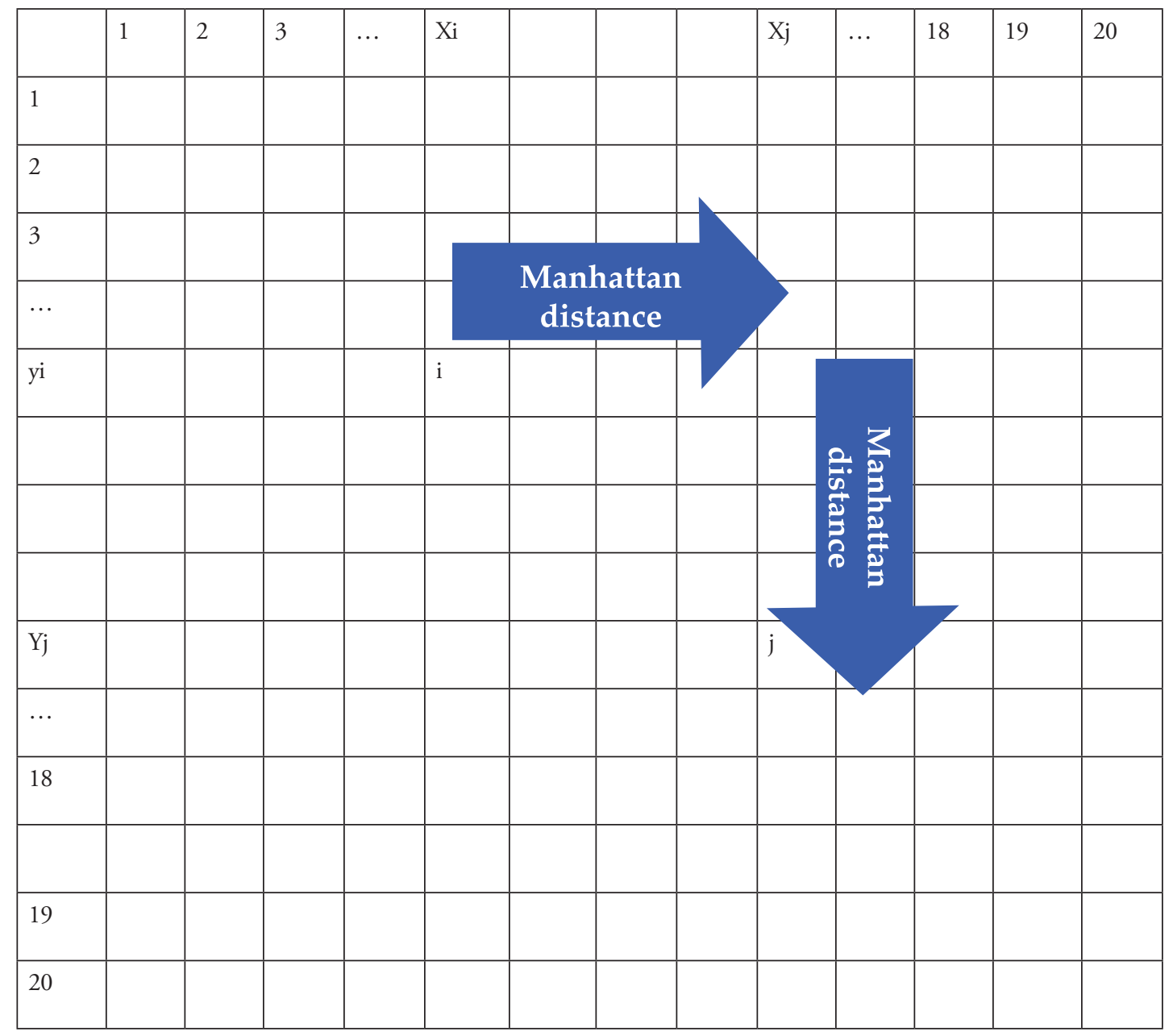

Figure 1. Conceptual diagram of simulated city structure and Manhattan-based travel times 

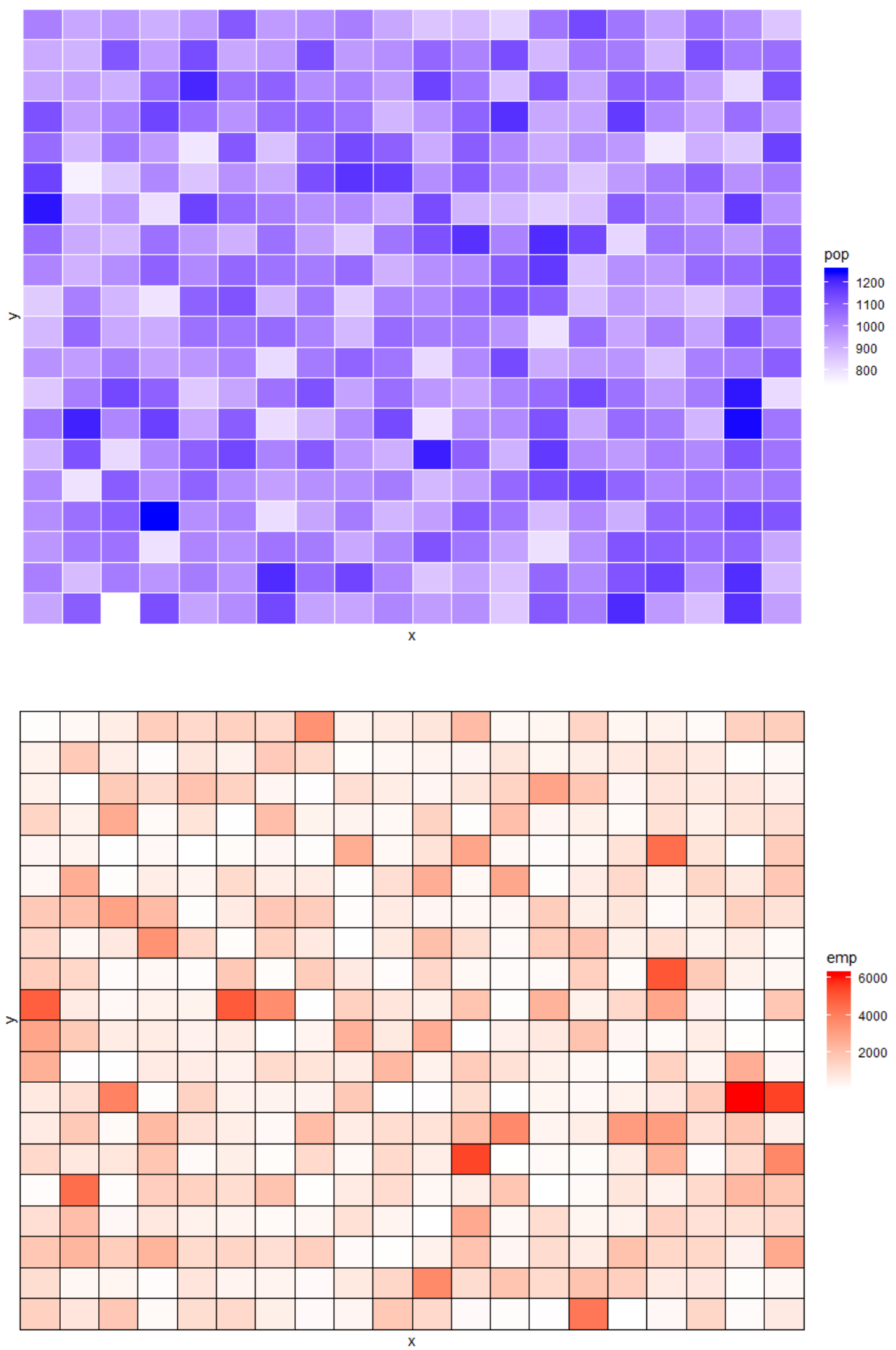

Figure 2. Population and employment densities by zone for a sample simulated city

Travel times are determined through a Manhattan travel distance (assuming travel only takes place parallel to the $\mathrm{x}$ and $\mathrm{y}$ axes along a rectilinear grid) with some randomization added to create a more naturalistic distribution of travel times. The travel time between adjacent districts (not including diagonally adjacent districts) is set at 5 minutes, and 5 minutes are added for each additional horizontal or 
vertical movement. In addition, each zone-to-zone travel times are perturbed by a random integer value between -2 and 2 , so that any positive integer minutes of travel times is possible between 3 minutes as the minimum and 192 minutes as the maximum.

The formula for travel time between zones $i$ and $j$ is as follows:

$$
t_{i j}=5 *\left[a b s\left(x_{i}-x_{j}\right)+a b s\left(y_{i}-y_{j}\right)\right]+\operatorname{rand}(-2,-1,-0,1,2)
$$

Where $t_{i j}$ is the travel time between zones in minutes $x_{i}$ and $x_{j}$ are the column numbers and $y_{i}$ and $\mathrm{y}_{j}$ are the row numbers of the zones $i$ and $j$ respectively. The rand () function indicates that one of the values in the list is selected at random. Note that travel times are not necessarily symmetrical, i.e., $t_{\mathrm{ij}}$ is not necessarily equal to $t_{\mathrm{ij}}$.

Intrazonal travel times are then computed as $1 / 2$ the average travel time to the three closest zones, rounded to the nearest minute. Therefore, intrazonal travel times range from a possible minimum of 2 minutes to a possible maximum of 4 minutes.

Figure 3 displays the population-weighted zone-to-zone travel time distribution resulting from the above method.

\section{Distribution of Zone-to-Zone Travel Times}

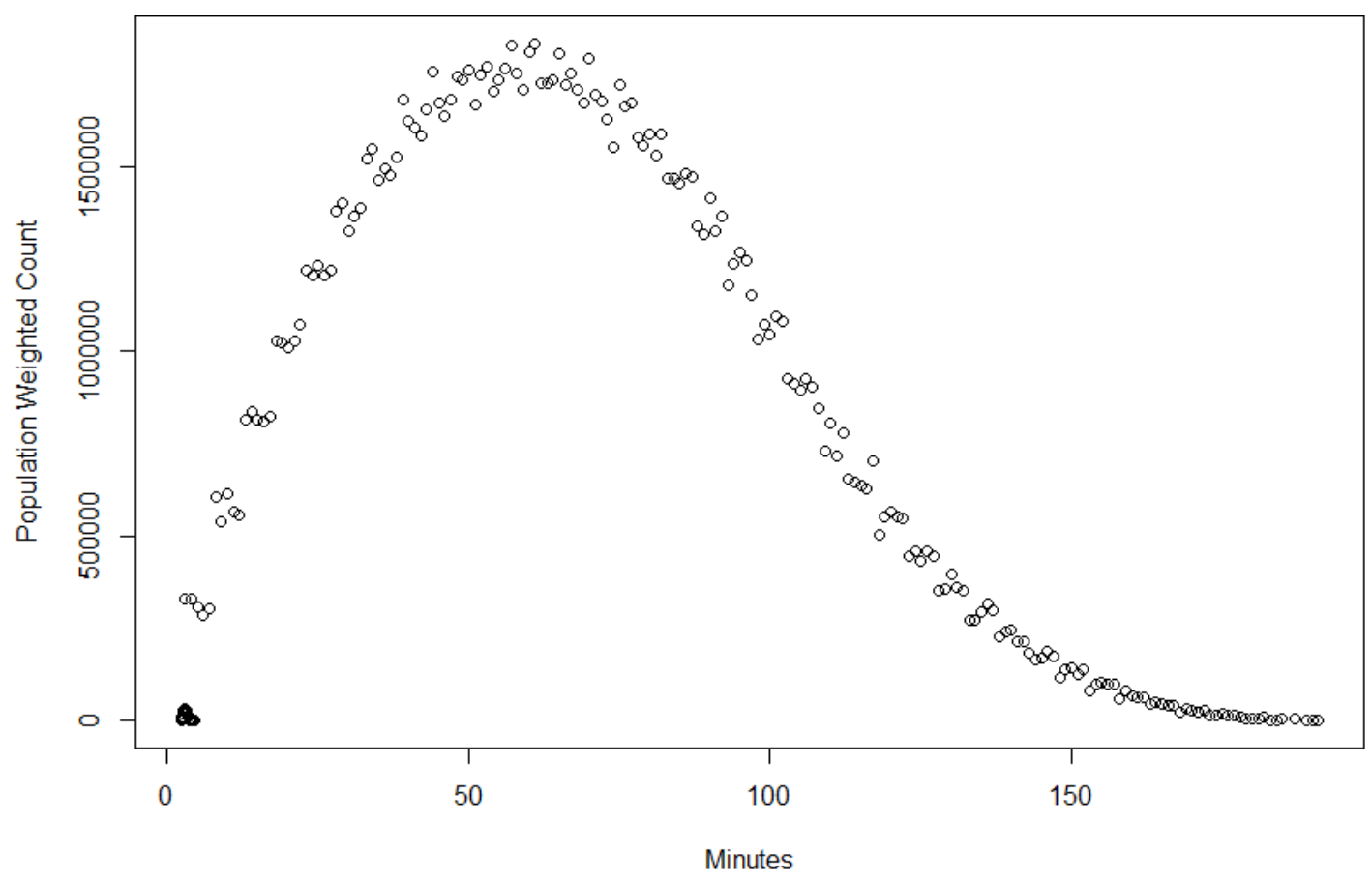

Figure 3: Population-weighted distribution of interzonal travel times in minutes

The true impedance $(\beta)$ is set at the beginning of the analysis with the hope of recovering it via the proposed method. This true impedance is then used to generate a series of observed zone-to-zone flows for each simulated city based on a doubly-constrained spatial interaction model. The $A_{\mathrm{i}}$ origin balancing factor and the $B_{\mathrm{j}}$ destination balancing factor are calculated based upon the true value of impedance $(\beta)$. Then the observed flow between zones $\mathrm{i}$ and $\mathrm{j}$ is calculated based upon a doubly-constrained spatial interaction model with a negative exponential impedance function: 


$$
F_{i j}=A_{i} B_{j} o_{i} d_{j} e^{-\beta * t_{i j}}
$$

Where $F_{\mathrm{ij}}$ is the flow of workers from zone $i$ into jobs in zone $j, A_{i}$ is the origin balancing factor for zone $i, B_{\mathrm{j}}$ is the destination balancing factor for zone $j, o_{\mathrm{i}}$ is the number of workers in zone $i, d_{j}$ is the number of jobs in zone $j$, and $t_{i j}$ is the travel time from zone $i$ to zone $j$.

Then based on these flows $F_{i j}$ between zones, a median travel time is recovered.

Finally based upon this median travel time, the proposed methodology is applied and an estimated impedance $\beta_{M E D}$ is recovered.

This median calibration method is then compared to two alternative fast-calibration methods. The first is the method proposed by Osth et. al. (2014) expressed in equation (13) above. And the second method is based on the observed trip length distribution of worker flows.

The observed trip length distribution method (TLD) works as follows. If $y_{t}$ is the number of travelers observed with travel time $t$, then a $\log$-linear model is used to estimate the impedance coefficient $\beta_{T L D}$ :

$$
\begin{aligned}
& y_{t}=\text { of travelers with travel time } t, t \geq 3 \text { and } y_{t}>0 \\
& \log \left(y_{t}\right)=\alpha+\beta_{T L D} * t
\end{aligned}
$$

In addition, I also tested the median calibration method with a power impedance function. The procedure is the same as above, except that the calibration of flows is based upon a power function:

$$
F_{i j}=A_{i} B_{j} o_{i} d_{j} t_{i j}^{-\beta}
$$

Then from this, the travel time distribution and median travel time are derived. Based upon median travel time, an estimated impedance coefficient $\beta_{M E D}$ is estimated as described above. This impedance estimate is then compared with a calibration based upon a trip length distribution method, which involves the following two formulae

$$
\begin{aligned}
& y_{t}=\text { of travelers with travel time } t, t \geq 3 \text { and } y_{t}>0 \\
& \log \left(y_{t}\right)=\alpha+\beta_{T L D} * \log (t)
\end{aligned}
$$

\section{$6 \quad$ Results}

The estimates of the power impedance parameter based on median calibration range in average error from are below $12.1 \%$ for all impedance values in the range of $0.5-2.0$ and below $5.0 \%$ for the values between $0.8-1.8$. The variation in such errors across simulations is small, typically $1-2 \%$, which means that the median calibration method provides robust results across a range of city geographies. See Table 1 for full results on calibrating for the power function. In comparison, the Trip Length Distribution Method has very large error estimates - in all cases higher than 26\% error, and in many cases more than $100 \%$ in error; that is the estimated impedance parameter is more than double its true value using this method for impedance values in the 0.5-0.8 range. Although the Trip Length Distribution method of calibration is widely used, the simulation results here indicate that it is not a reliable method for calibrating power-based gravity spatial interaction models.

For the exponential impedance function, parameter estimate errors are less than $10 \%$ for impedance values between 0.02 and 0.13 , between $10-20 \%$ for impedance values $0.14-0.21$, and between $20-30 \%$ for impedance values of 0.22 and above. See Table 2 for full results on calibrating for the expo- 
nential function. The median method is almost always more accurate than the alternative Osth method and the trip length distribution method, except in the case where true impedance $=0.07$ or 0.08 , in which case the trip length distribution method is slightly more accurate. The small standard errors in parameter estimation error for the median method show that the process yields very similar values across different simulated cities. The negative exponential impedance parameter estimates based on the median calibration method have a range of errors that depend highly upon the true impedance value. Indeed, the amount of error in the median impedance estimate appears to grow systematically with the value of the true impedance.

How accurate are sample estimates of median travel times? This depends upon the underlying travel time distribution, but the simulations here are used to examine this question. Table 3 and Table 4 illustrate some results obtained from the simulations on how accurately sample medians reflect true median values. Interestingly the accuracy of the sample median depends upon the impedance as well as the sample size. For example, for samples of 200 , a precise median is only obtained $9 \%$ of the time for impedance values $=0.01$, but is obtained $55 \%$ of the time for impedance values $=0.30$. The accuracy of the true median increases as the true impedance value increases. The same pattern is observed for how often median estimates are within one minute of the correct value. Again for a sample of 200, the sample estimate is within 1 minute of the true value $34 \%$ of the time for impedance $=0.01$ but $98 \%$ of the time for impedance $=0.30$. Therefore, there is no universal rule about how large a sample must be to obtain an accurate median. Assuming a typical impedance value of 0.10 , a sample size of 250 will result in a median estimate within 1 minute of the true value $85 \%$ of the time. So clearly large samples are to be preferred where possible.

Table 1. Impedance estimates for power impedance functional form

\begin{tabular}{|r|r|r|r|r|r|r|}
\hline $\begin{array}{c}\text { True Impedance } \\
\text { Value }\end{array}$ & $\begin{array}{c}\text { Mean Median } \\
\text { Estimate }\end{array}$ & $\begin{array}{c}\text { Mean TLD } \\
\text { Estimate }\end{array}$ & $\begin{array}{c}\text { Mean Error } \\
\text { in Median } \\
\text { Estimate }\end{array}$ & $\begin{array}{c}\text { Standard Deviation } \\
\text { of Error in Median } \\
\text { Estimate }\end{array}$ & $\begin{array}{c}\text { Mean Median } \\
\text { MLD Estimate }\end{array}$ & $\begin{array}{c}\text { Mravel Time in } \\
\text { Minutes }\end{array}$ \\
\hline 0.5 & 0.44 & 1.42 & $12.1 \%$ & $2.3 \%$ & $184.2 \%$ & 52.8 \\
\hline 0.6 & 0.54 & 1.50 & $9.6 \%$ & $1.7 \%$ & $150.4 \%$ & 49.9 \\
\hline 0.7 & 0.64 & 1.59 & $8.0 \%$ & $1.4 \%$ & $126.8 \%$ & 46.8 \\
\hline 0.8 & 0.76 & 1.67 & $5.4 \%$ & $1.3 \%$ & $108.4 \%$ & 43.2 \\
\hline 0.9 & 0.86 & 1.75 & $5.0 \%$ & $0.8 \%$ & $94.1 \%$ & 40.0 \\
\hline 1.0 & 0.97 & 1.83 & $3.5 \%$ & $0.9 \%$ & $83.1 \%$ & 36.1 \\
\hline 1.1 & 1.06 & 1.91 & $3.6 \%$ & $1.0 \%$ & $73.9 \%$ & 32.7 \\
\hline 1.2 & 1.16 & 1.99 & $3.2 \%$ & $0.4 \%$ & $66.0 \%$ & 29.0 \\
\hline 1.3 & 1.27 & 2.07 & $2.5 \%$ & $0.7 \%$ & $59.4 \%$ & 25.1 \\
\hline 1.4 & 1.36 & 2.15 & $2.9 \%$ & $0.6 \%$ & $53.5 \%$ & 21.9 \\
\hline 1.5 & 1.45 & 2.22 & $3.5 \%$ & $0.8 \%$ & $48.2 \%$ & 18.8 \\
\hline 1.6 & 1.54 & 2.29 & $3.8 \%$ & $0.6 \%$ & $43.4 \%$ & 15.9 \\
\hline 1.7 & 1.63 & 2.36 & $4.4 \%$ & $1.2 \%$ & $38.8 \%$ & 13.4 \\
\hline 1.8 & 1.71 & 2.42 & $5.0 \%$ & $1.0 \%$ & $34.3 \%$ & 11.3 \\
\hline 1.9 & 1.77 & 2.47 & $6.8 \%$ & $1.0 \%$ & $30.2 \%$ & 9.8 \\
\hline 2 & 1.85 & 2.52 & $7.4 \%$ & $1.5 \%$ & $26.2 \%$ & 8.3 \\
\hline
\end{tabular}

Note: Means and standard deviations are taken over a sample of 50 simulated cities.

TLD is short for the Trip Length Distribution method. 
Table 2. Impedance estimates for exponential functional form

\begin{tabular}{|c|c|c|c|c|c|c|c|c|}
\hline $\begin{array}{l}\text { True } \\
\text { Impedance } \\
\text { Value }\end{array}$ & $\begin{array}{l}\text { Mean } \\
\text { Median } \\
\text { Estimate }\end{array}$ & $\begin{array}{l}\text { Mean } \\
\text { Osth-based } \\
\text { Estimate }\end{array}$ & $\begin{array}{l}\text { Mean Trip } \\
\text { Length } \\
\text { Distribution } \\
\text { Estimate }\end{array}$ & $\begin{array}{l}\text { Median } \\
\text { Estimate } \\
\text { Mean } \\
\text { Error }\end{array}$ & $\begin{array}{l}\text { Median } \\
\text { Estimate } \\
\text { Std. Dev. } \\
\text { Error }\end{array}$ & $\begin{array}{l}\text { Osth } \\
\text { Estimate } \\
\text { Mean } \\
\text { Error }\end{array}$ & $\begin{array}{l}\text { TLD } \\
\text { Mean } \\
\text { Error }\end{array}$ & $\begin{array}{l}\text { Mean } \\
\text { Median } \\
\text { Travel } \\
\text { Time in } \\
\text { Minutes }\end{array}$ \\
\hline 0.01 & 0.008 & 0.013 & 0.032 & $16.2 \%$ & $3.1 \%$ & $28.8 \%$ & $222.6 \%$ & 53.8 \\
\hline 0.02 & 0.018 & 0.016 & 0.039 & $7.9 \%$ & $1.0 \%$ & $21.2 \%$ & $96.8 \%$ & 44.0 \\
\hline 0.03 & 0.029 & 0.019 & 0.046 & $3.6 \%$ & $0.8 \%$ & $35.9 \%$ & $53.8 \%$ & 36.0 \\
\hline 0.04 & 0.039 & 0.023 & 0.053 & $2.0 \%$ & $1.1 \%$ & $42.3 \%$ & $31.4 \%$ & 30.1 \\
\hline 0.05 & 0.048 & 0.027 & 0.059 & $3.3 \%$ & $0.6 \%$ & $46.6 \%$ & $17.8 \%$ & 26.0 \\
\hline 0.06 & 0.058 & 0.031 & 0.065 & $2.8 \%$ & $2.6 \%$ & $48.6 \%$ & $8.7 \%$ & 22.5 \\
\hline 0.07 & 0.067 & 0.035 & 0.072 & $4.2 \%$ & $1.1 \%$ & $50.4 \%$ & $2.5 \%$ & 20.0 \\
\hline 0.08 & 0.076 & 0.039 & 0.078 & $5.2 \%$ & $1.1 \%$ & $51.9 \%$ & $2.3 \%$ & 18.0 \\
\hline 0.09 & 0.086 & 0.043 & 0.085 & $4.5 \%$ & $1.3 \%$ & $52.0 \%$ & $6.0 \%$ & 16.1 \\
\hline 0.10 & 0.094 & 0.047 & 0.091 & $6.4 \%$ & $2.9 \%$ & $53.0 \%$ & $8.8 \%$ & 14.8 \\
\hline 0.11 & 0.103 & 0.051 & 0.098 & $6.4 \%$ & $4.0 \%$ & $53.3 \%$ & $11.3 \%$ & 13.5 \\
\hline 0.12 & 0.109 & 0.054 & 0.104 & $8.9 \%$ & $3.7 \%$ & $54.8 \%$ & $13.3 \%$ & 12.8 \\
\hline 0.13 & 0.118 & 0.058 & 0.110 & $9.0 \%$ & $2.4 \%$ & $55.2 \%$ & $15.1 \%$ & 11.9 \\
\hline 0.14 & 0.126 & 0.063 & 0.117 & $10.2 \%$ & $2.1 \%$ & $55.3 \%$ & $16.6 \%$ & 11.1 \\
\hline 0.15 & 0.134 & 0.067 & 0.123 & $10.6 \%$ & $3.6 \%$ & $55.0 \%$ & $18.1 \%$ & 10.3 \\
\hline 0.16 & 0.139 & 0.070 & 0.129 & $13.2 \%$ & $3.0 \%$ & $56.2 \%$ & $19.5 \%$ & 9.9 \\
\hline 0.17 & 0.148 & 0.075 & 0.135 & $12.8 \%$ & $3.9 \%$ & $55.9 \%$ & $20.8 \%$ & 9.3 \\
\hline 0.18 & 0.151 & 0.077 & 0.140 & $16.0 \%$ & $2.6 \%$ & $57.4 \%$ & $22.0 \%$ & 9.1 \\
\hline 0.19 & 0.161 & 0.081 & 0.146 & $15.4 \%$ & $5.2 \%$ & $57.1 \%$ & $23.2 \%$ & 8.5 \\
\hline 0.2 & 0.166 & 0.084 & 0.151 & $17.1 \%$ & $4.6 \%$ & $58.0 \%$ & $24.4 \%$ & 8.3 \\
\hline 0.21 & 0.170 & 0.086 & 0.156 & $18.9 \%$ & $1.9 \%$ & $58.9 \%$ & $25.6 \%$ & 8.0 \\
\hline 0.22 & 0.174 & 0.088 & 0.161 & $20.7 \%$ & $4.6 \%$ & $59.9 \%$ & $26.7 \%$ & 7.9 \\
\hline 0.23 & 0.186 & 0.093 & 0.166 & $19.1 \%$ & $6.2 \%$ & $59.4 \%$ & $27.8 \%$ & 7.5 \\
\hline 0.24 & 0.189 & 0.095 & 0.171 & $21.2 \%$ & $5.7 \%$ & $60.5 \%$ & $28.9 \%$ & 7.3 \\
\hline 0.25 & 0.194 & 0.097 & 0.175 & $22.4 \%$ & $4.6 \%$ & $61.0 \%$ & $29.9 \%$ & 7.1 \\
\hline 0.26 & 0.203 & 0.104 & 0.180 & $21.8 \%$ & $5.7 \%$ & $60.0 \%$ & $30.9 \%$ & 6.7 \\
\hline 0.27 & 0.209 & 0.108 & 0.184 & $22.5 \%$ & $4.8 \%$ & $60.0 \%$ & $32.0 \%$ & 6.5 \\
\hline 0.28 & 0.212 & 0.110 & 0.188 & $24.2 \%$ & $4.0 \%$ & $60.6 \%$ & $32.9 \%$ & 6.3 \\
\hline 0.29 & 0.214 & 0.112 & 0.192 & $26.3 \%$ & $3.7 \%$ & $61.5 \%$ & $33.8 \%$ & 6.2 \\
\hline 0.3 & 0.216 & 0.113 & 0.196 & $28.1 \%$ & $3.5 \%$ & $62.3 \%$ & $34.7 \%$ & 6.2 \\
\hline
\end{tabular}

Note: Means and standard deviations are taken over a sample of 50 simulated cities.

TLD is short for the Trip Length Distribution method. 
Table 3. Percentage of time exact median travel time is obtained from sample based on impedance value and sample size $(\mathrm{N})$

\begin{tabular}{|c|c|c|c|c|c|}
\hline Impedance & $\mathrm{N}=50$ & $N=100$ & $N=150$ & $\mathrm{~N}=\mathbf{2 0 0}$ & $\mathrm{N}=250$ \\
\hline 0.01 & $3 \%$ & $5 \%$ & $7 \%$ & $9 \%$ & $10 \%$ \\
\hline 0.02 & $4 \%$ & $6 \%$ & $8 \%$ & $10 \%$ & $12 \%$ \\
\hline 0.03 & $4 \%$ & $7 \%$ & $9 \%$ & $11 \%$ & $13 \%$ \\
\hline 0.04 & $5 \%$ & $8 \%$ & $11 \%$ & $15 \%$ & $17 \%$ \\
\hline 0.05 & $6 \%$ & $10 \%$ & $13 \%$ & $17 \%$ & $20 \%$ \\
\hline 0.06 & $7 \%$ & $11 \%$ & $15 \%$ & $19 \%$ & $23 \%$ \\
\hline 0.07 & $8 \%$ & $13 \%$ & $18 \%$ & $22 \%$ & $26 \%$ \\
\hline 0.08 & $10 \%$ & $17 \%$ & $23 \%$ & $28 \%$ & $32 \%$ \\
\hline 0.09 & $9 \%$ & $17 \%$ & $22 \%$ & $27 \%$ & $32 \%$ \\
\hline 0.1 & $11 \%$ & $19 \%$ & $26 \%$ & $30 \%$ & $34 \%$ \\
\hline 0.11 & $13 \%$ & $23 \%$ & $30 \%$ & $35 \%$ & $38 \%$ \\
\hline 0.12 & $15 \%$ & $25 \%$ & $33 \%$ & $39 \%$ & $43 \%$ \\
\hline 0.13 & $12 \%$ & $21 \%$ & $28 \%$ & $33 \%$ & $37 \%$ \\
\hline 0.14 & $14 \%$ & $24 \%$ & $31 \%$ & $37 \%$ & $42 \%$ \\
\hline 0.15 & $17 \%$ & $28 \%$ & $36 \%$ & $40 \%$ & $45 \%$ \\
\hline 0.16 & $18 \%$ & $30 \%$ & $38 \%$ & $44 \%$ & $49 \%$ \\
\hline 0.17 & $22 \%$ & $34 \%$ & $42 \%$ & $47 \%$ & $49 \%$ \\
\hline 0.18 & $22 \%$ & $36 \%$ & $46 \%$ & $53 \%$ & $60 \%$ \\
\hline 0.19 & $24 \%$ & $38 \%$ & $44 \%$ & $49 \%$ & $51 \%$ \\
\hline 0.2 & $27 \%$ & $40 \%$ & $50 \%$ & $55 \%$ & $58 \%$ \\
\hline 0.21 & $27 \%$ & $42 \%$ & $53 \%$ & $60 \%$ & $65 \%$ \\
\hline 0.22 & $26 \%$ & $40 \%$ & $50 \%$ & $56 \%$ & $61 \%$ \\
\hline 0.23 & $22 \%$ & $36 \%$ & $44 \%$ & $49 \%$ & $52 \%$ \\
\hline 0.24 & $22 \%$ & $35 \%$ & $44 \%$ & $50 \%$ & $54 \%$ \\
\hline 0.25 & $21 \%$ & $34 \%$ & $41 \%$ & $47 \%$ & $51 \%$ \\
\hline 0.26 & $22 \%$ & $35 \%$ & $43 \%$ & $48 \%$ & $52 \%$ \\
\hline 0.27 & $23 \%$ & $36 \%$ & $44 \%$ & $49 \%$ & $51 \%$ \\
\hline 0.28 & $23 \%$ & $38 \%$ & $45 \%$ & $49 \%$ & $50 \%$ \\
\hline 0.29 & $24 \%$ & $39 \%$ & $48 \%$ & $53 \%$ & $57 \%$ \\
\hline 0.3 & $25 \%$ & $40 \%$ & $50 \%$ & $55 \%$ & $59 \%$ \\
\hline
\end{tabular}

For exponential impedance funtion 
Table 4. Percentage of time sample median estimate is $=+/-1$ minute based on impedance value and sample size $(\mathrm{N})$

\begin{tabular}{|c|c|c|c|c|c|}
\hline Impedance & $\mathrm{N}=50$ & $\mathrm{~N}=100$ & $\mathrm{~N}=150$ & $\mathrm{~N}=\mathbf{2 0 0}$ & $\mathrm{N}=\mathbf{2 5 0}$ \\
\hline 0.01 & $16 \%$ & $23 \%$ & $29 \%$ & $34 \%$ & $37 \%$ \\
\hline 0.02 & $19 \%$ & $26 \%$ & $32 \%$ & $38 \%$ & $43 \%$ \\
\hline 0.03 & $21 \%$ & $29 \%$ & $36 \%$ & $43 \%$ & $48 \%$ \\
\hline 0.04 & $25 \%$ & $35 \%$ & $43 \%$ & $51 \%$ & $55 \%$ \\
\hline 0.05 & $27 \%$ & $39 \%$ & $48 \%$ & $55 \%$ & $61 \%$ \\
\hline 0.06 & $32 \%$ & $44 \%$ & $54 \%$ & $62 \%$ & $68 \%$ \\
\hline 0.07 & $36 \%$ & $51 \%$ & $61 \%$ & $69 \%$ & $75 \%$ \\
\hline 0.08 & $40 \%$ & $56 \%$ & $66 \%$ & $75 \%$ & $81 \%$ \\
\hline 0.09 & $42 \%$ & $57 \%$ & $68 \%$ & $76 \%$ & $81 \%$ \\
\hline 0.1 & $46 \%$ & $64 \%$ & $74 \%$ & $81 \%$ & $85 \%$ \\
\hline 0.11 & $52 \%$ & $69 \%$ & $80 \%$ & $85 \%$ & $89 \%$ \\
\hline 0.12 & $53 \%$ & $71 \%$ & $81 \%$ & $87 \%$ & $91 \%$ \\
\hline 0.13 & $53 \%$ & $72 \%$ & $82 \%$ & $88 \%$ & $91 \%$ \\
\hline 0.14 & $56 \%$ & $74 \%$ & $84 \%$ & $90 \%$ & $93 \%$ \\
\hline 0.15 & $61 \%$ & $77 \%$ & $85 \%$ & $90 \%$ & $92 \%$ \\
\hline 0.16 & $64 \%$ & $82 \%$ & $90 \%$ & $94 \%$ & $96 \%$ \\
\hline 0.17 & $70 \%$ & $84 \%$ & $91 \%$ & $94 \%$ & $95 \%$ \\
\hline 0.18 & $72 \%$ & $89 \%$ & $95 \%$ & $98 \%$ & $99 \%$ \\
\hline 0.19 & $72 \%$ & $88 \%$ & $94 \%$ & $96 \%$ & $98 \%$ \\
\hline 0.2 & $74 \%$ & $90 \%$ & $95 \%$ & $98 \%$ & $99 \%$ \\
\hline 0.21 & $74 \%$ & $90 \%$ & $95 \%$ & $98 \%$ & $99 \%$ \\
\hline 0.22 & $73 \%$ & $89 \%$ & $94 \%$ & $97 \%$ & $98 \%$ \\
\hline 0.23 & $74 \%$ & $90 \%$ & $95 \%$ & $98 \%$ & $99 \%$ \\
\hline 0.24 & $74 \%$ & $90 \%$ & $96 \%$ & $98 \%$ & $99 \%$ \\
\hline 0.25 & $74 \%$ & $90 \%$ & $95 \%$ & $97 \%$ & $98 \%$ \\
\hline 0.26 & $75 \%$ & $89 \%$ & $95 \%$ & $97 \%$ & $98 \%$ \\
\hline 0.27 & $76 \%$ & $89 \%$ & $94 \%$ & $96 \%$ & $97 \%$ \\
\hline 0.28 & $77 \%$ & $90 \%$ & $94 \%$ & $96 \%$ & $97 \%$ \\
\hline 0.29 & $79 \%$ & $92 \%$ & $96 \%$ & $97 \%$ & $98 \%$ \\
\hline 0.3 & $80 \%$ & $92 \%$ & $97 \%$ & $98 \%$ & $99 \%$ \\
\hline
\end{tabular}

For exponential impedance funtion

\section{Discussion}

The median calibration method produces reasonably accurate estimates of the true impedance parameter for both exponential and power impedance functions. In both cases, it outperforms the accuracy of comparable fast methods such as the method proposed by Osth et. al. (2014) or the commonly used method of calibrating to trip length distribution curve (TLD method).

Although the proposed impedance parameter estimation method generally creates accurate esti- 
mates for common values of impedance, it is notably less accurate for the negative exponential function for high levels of true impedance. For high values of impedance, the existing spatial structure may not permit sufficiently short trip lengths to reflect traveler preferences. Trip lengths hit a minimum duration due to the limitations of spatial structure and origin and destination constraints in the doublyconstrained context. Therefore, the observed median travel times may not fully reflect the subjectively experienced resistance to traveling further distances.

Using median values to estimate impedance values for gravity accessibility reduces the data requirements and technical expertise required for accessibility calculations. The formulas above do not require access to specialized travel demand modeling software and can in concept be calculated with standard spreadsheet tools.

The accuracy of the sample median depends highly both on the sample size and the underlying travel time distribution. That travel time distribution in turn depends upon the true value of impedance. Median estimates become more accurate for higher true values of impedance. In general, it appears that sample sizes of 200 or greater are desirable for obtaining a reasonably accurate estimate of median travel time. Although median travel time is not currently reported in US Census American Community Survey data on commuting, the data already collected could readily provide median commute travel times for a variety of geographic areas and populations — cities, counties, different income ranges, travel modes and genders — - on an annual basis.

Since median travel times can be estimated from relatively small samples ( $\mathrm{n} \sim 200)$, existing census and other public survey sources can be used to provide the necessary travel behavior information to calibrate impedance parameters. As interest in the deployment of accessibility metrics for transportation analysis spreads, the availability of new, simplified and streamlined methods for calibrating gravity-based accessibility measures is a welcome development.

Estimating impedance values based on median travel time alone also allows for greater nuance in the calculation of how accessibility varies by population group, by trip purpose, and/or by mode. For example, the impedance parameters for accessibility to work for low-income populations and high-income populations can be readily calculated separately via this method. So if high-income populations drive further to work than low-income populations do, this greater constraint on low-income populations could be taken into account. Likewise, the calculation of impedance parameters for less-visited destinations, such as shopping areas or parks, can be calibrated with smaller samples of traveler behavior using this method.

\section{Conclusions}

This paper presents a method for calibrating the impedance parameter of a spatial interaction model using only the median travel time as the measure of observed traveler behavior, alongside complete information about the spatial structure and interzonal travel times. The proposed method allows for expedited calibration of doubly-constrained spatial interaction models and allows the calibration of spatial interaction models with smaller samples of travel behavior data. The method does not require expensive software and can be implemented with little technical capacity, including via spreadsheet software. The method allows for the customized calibration of spatial interaction models for specific population segments, travel modes, and destination types.

Using simulated city data with worker counts and job counts detailed by zone and interzonal travel times defined, estimated impedance values using this method are compared with the "true" apriori known impedance parameters. For power impedance functions, errors were consistently below $12.1 \%$ and typically below $10 \%$. For negative exponential impedance functions, errors ranged to as high as $28.1 \%$ but were lower than $10 \%$ for a significant range of impedance values $(0.02-0.13)$. The proposed 
median method consistently produced more accurate estimates of the true parameter value than alternative fast methods, such as calibrating to the Trip Length Distribution (TLD). As a result, the method appears viable for practical application in the calibration and implementation of gravity-based accessibility measures.

Reducing the data requirements and computational burden associated with calculating gravity accessibility measures should make such measures more usable for a wider swath of transportation analysts and professionals. Cumulative opportunity measures are currently much more widely used than gravity accessibility measures in applied realms (Boisjoly \& El-Geneidy, 2017a; Virginia Department of Transportation, 2016). However, the problem with cumulative opportunity measures is that they rely upon arbitrary cut-offs regarding which destinations are considered. The proposed method of median calibration here relies upon observed traveler behavior and the spatial structure of the analysis area under consideration. Therefore, the proposed method is strongly rooted in the specific environment under analysis. Further, because of the modest data requirements for median calibration (typically $n=200$ ), impedance values can be tailored for a range of situations, i.e., for varying modes, travel purposes, targeted traveler groups, and/or specific times of day. 


\section{References}

Batty, M., \& Sikdar, P. K. (1982). Spatial aggregation in gravity models: An information-theoretic framework. Environment and Planning A, 14(3), 377-405. doi.org/10.1068/a140377

Batty, M., \& Mackie, S. (1972). The calibration of gravity, entropy and related models of spatial interaction. Environment and Planning A, 4(2), 205-233. doi.org/10.1068/a040205

Boisjoly, G., \& El-Geneidy, A. M. (2017a). How to get there? A critical assessment of accessibility objectives and indicators in metropolitan transportation plans. Transport Policy, 55, 38-50. doi. org/10.1016/j.tranpol.2016.12.011

Boisjoly, G., \& El-Geneidy, A. M. (2017b). The insider: A planners' perspective on accessibility. In Transportation Research Board 96th Annual Meeting (pp. 1-19). Washington, DC: Transportation Research Board.

de Vries, J. J., Nijkamp, P., \& Rietveld, P. (2009). Exponential or power distance-decay for commuting? An alternative specification. Environment and Planning A, 41(2), 461-480. doi.org/10.1068/ a39369

Dennett, A. (2012). Estimating flows between geographical locations: Get me started in spatial interaction modelling (No. Paper 181). UCL Working Papers Series (Vol. 44). London: Center for Advanced Spatial Analysis, University College. Retrieved from http://citeseerx.ist.psu.edu/viewdoc/download? doi=10.1.1.226.620\&rep=rep1\&type=pdf

Diplock, G., \& Openshaw, S. (2010). Using simple genetic algorithms to calibrate spatial interaction models. Geographical Analysis, 28(3), 262-279. doi.org/10.1111/j.1538-4632.1996.tb00934.x

Feldman, O., Forero-Martinez, J., \& Coombe, D. (2012). Alternative gravity modelling approaches for trip matrix. In Association for European Transport and Contributors (pp. 1-17). Glasgow, UK: Conference Papers 2012. Retrieved from https://aetransport.org/en-gb/past-etc-papers/conferencepapers-2012

Fotheringham, A. S. (1983). A new set of spatial-interaction models: The theory of competing destinations. Environment and Planning A, 15(1), 15-36. doi.org/10.1177/0308518X8301500103

Fotheringham, A. S., \& O'Kelly, M. E. (1989). Spatial interaction models: Formulations and applications. Dordrecht, the Netherlands: Kluwer Academic.

Goncalves, M. B., \& Ulyssea-Neto, I. (1993). The development of a new gravity-opportunity model for trip distribution. Environment and Planning A, 25(1960), 817-826.

Gray, R. H., \& Sen, A. K. (1983). Estimating gravity model parameters: A simplified approach based on the odds ratio. Transportation Research Part B: Methodological, 17(2), 117-131.

Gutman, J., \& Tomer, A. (2016). Developing a common narrative on urban accessibility: Overview. Washington DC: Brookings. Retrieved from https://www.brookings.edu/wp-content/uploads/2017/01/ overview-digital.pdf

Hayes, M. C., \& Wilson, A. G. (1971). Spatial interaction. Socio-Economic Planning Sciences, 5, 73-95. doi.org/https://doi.org/10.1016/0038-0121(71)90042-5

Horner, M. W., \& Murray, A. T. (2002). Excess commuting and the modifiable areal unit problem. Urban Studies, 39(1), 131-139. doi.org/10.1080/0042098022009911

Hyman, G. M. (1969). The calibration of trip distribution models. Environment and Planning A: Economy and Space, 1(1), 105-112. doi.org/10.1068/a010105

Krizek, K. J. (2010). Measuring accessibility: Prescriptions for performance measures of the creative and sustainable city. International Journal of Sustainable Development, 13(1/2), 149. doi.org/10.1504/ ijsd.2010.035105

Lenormand, M., Bassolas, A., \& Ramasco, J. J. (2016). Systematic comparison of trip distribution laws and models. Journal of Transport Geography, 51, 158-169. doi.org/10.1016/j.jtrangeo.2015.12.008 
Melhorado, A. M. C., Demirel, H., Kompil, M., Navajas, E., \& Panayotis, C. (2016). The impact of measuring internal travel distances on self-potentials and accessibility. European Journal of Transport and Infrastructure Research, 16(2), 300-318. doi.org/https://doi.org/10.18757/ejtir.2016.16.2.3139

Murat, C. H. (2010). Sample size needed for calibrating trip distribution and behavior of the gravity model. Journal of Transport Geography, 18(1), 183-190. doi.org/10.1016/j.jtrangeo.2009.05.013

Ortuzar, J. de D., \& Willumsen, L. G. (2011). Modeling transport (4th ed.). Chichester: John Wiley $\&$ Sons.

Osth, J., Reggiani, A., \& Galiazzo, G. (2014). Novel methods for the estimation of cost-distance decay in potential accessibility models. In A. Condeco-Melhorado, A. Reggiani, \& J. Gutierrez (Eds.), Accessibility and spatial interaction (pp. 15-37). Cheltenham, UK: Edward Elgar Publishing Ltd.

Piovani, D., Arcaute, E., Uchoa, G., Wilson, A. G., \& Batty, M. (2018). Measuring accessibility using gravity and radiation models. Royal Society Open Science. doi.org/10.1098/rsos. 171668

Reggiani, A., Bucci, P., \& Russo, G. (2011). Accessibility and impedance forms: Empirical applications to the German commuting network. International Regional Science Review, 34(2), 230-252. doi. org/10.1177/0160017610387296

Sen, A., \& Pruthi, R. K. (1983). Least-squares calibration of the gravity model when intrazonal flows are unknown. Environment and Planning A, 15(2), 1545-1550.

Simini, F., González, M. C., Maritan, A., \& Barabási, A.-L. (2012). A universal model for mobility and migration patterns. Nature, 484(7392), 96. doi.org/doi:10.1038/nature10856

Stępniak, M., \& Jacobs-Crisioni, C. (2017). Reducing the uncertainty induced by spatial aggregation in accessibility and spatial interaction applications. Journal of Transport Geography, 61(April), 17-29. doi.org/10.1016/j.jtrangeo.2017.04.001

Stouffer, S. A. (1940). Intervening opportunities: A theory relating mobility and distance. American Sociological Review, 5(6), 845-867. doi.org/10.2307/2084520

Taylor, P. J. (1971). Distance transformation and distance decay functions. Geographic Analysis, 3(3), 221-238.

Virginia Department of Transportation. (2016). Smart Scale Technical Guide. Richmond, VA: Virginia Department of Transportation.

Williams, I. (1976). A comparison of some calibration techniques for doubly constrained models with an exponential cost function. Transportation Research, 10(2), 91-104. doi.org/10.1016/00411647(76)90045-9

Wilson, A. G. (1971). A family of spatial interaction models and associated developments. Environment and Planning A, 3(1), 1-32. doi.org/10.1068/a030001

WSP Parsons Brinckerhoff. (2017). Southeast Florida Household Travel Survey. Miami, FL. Retrieved from http://www.fsutmsonline.net/images/uploads/md_lctr_2006/SEFlorida_HTS_Final_Report _2017_12_05.pdf 